\title{
Nuclear Size in Plasmodia of the True Slime Mould Didymium nigripes
}

\author{
By SYLVIA J. KERR* \\ Department of Zoology, University of Minnesota, \\ Minneapolis, Minnesota 55455, U.S.A.
}

(Accepted for publication I September 1970)

\begin{abstract}
SUMMAR Y
Nuclei of different diameters, containing different numbers of chromosomes, are found in all macroscopic plasmodia of the true slime mould Didymium nigripes. The relative proportions of small and large nuclei vary in different plasmodial clones. Over extended periods of time c 6 wild-type clones maintain a constant proportion of small nuclei whereas Y 2-I, a yellow mutant, does not. Nuclear fusion (syngamy) does not occur prior to plasmodium formation in this isolate; neither is it delayed until the plasmodium has formed. The rate of formation of large nuclei in plasmodia was calculated. When plasmodia with differing nuclear size ratios were fused, the observed shifts in the nuclear size ratio suggested the possibility that parasexual processes could exist in the isolate under study.
\end{abstract}

\section{INTRODUCTION}

During the course of its life cycle, the true slime mould Didymium nigripes, passes through several morphologically distinct phases. Uninucleate spores germinate to form uninucleate amoebae. After several days of amoeboid growth and division, plasmodia form and continue to grow until they attain macroscopic size. When starved, plasmodia differentiate into fruiting bodies; within these fruiting bodies cytoplasmic cleavage occurs and uninucleate spores are formed, thus completing the life cycle. In many true slime moulds, plasmodia are initiated when two amoebae fuse; thus spores and amoebae are haploid, and plasmodia are diploid. The isolate of $D$. nigripes under study differs from a typical slime mould in that plasmodia form without prior amoebal or nuclear fusion: the plasmodia and amoebae both possess the same ploidy level (S. Kerr, I968a,b).

Phase contrast microscope observations of young plasmodia, and of smear preparations of pieces of older plasmodia which have grown to macroscopic size, have shown that mitotic division within a single plasmodium is an approximately synchronous process. Accordingly, it is logical to assume that all nuclei in a given plasmodium are in the same stage of the cell cycle at a given time; this has been demonstrated in a closely related slime mould, Physarum polycephalum (Nygaard, Guttes \& Rusch, 1960). Despite synchrony of the cell cycle, young plasmodia occasionally contain nuclei of different diameters. By the time a plasmodium has grown to macroscopic size, nuclei of two size classes are invariably present, and occasional nuclei of extraordinary size are also encountered.

* Present address: Department of Biology, Augsburg College, Minneapolis, Minnesota 55404. 
Chromosome counts in the strains of Didymium nigripes under investigation have made it possible to identify the approximate chromosome number of each of these size classes of nuclei (S. Kerr, I $968 a, b$ ). In effect, a plasmodium of one of these strains possesses a population of nuclei with approximately 32 to 33 chromosomes (small nuclei), nuclei with 64 to 66 chromosomes (large nuclei), and nuclei with over I00 chromosomes. The same studies showed that the proportion of small nuclei in populations of young plasmodia (with 2 to 16 nuclei) was not significantly different from the proportion of small nuclei in the populations of amoebae from which those plasmodia were derived. Thus no obvious change in ploidy level of nuclei occurs at the time of plasmodium formation.

The investigations reported here represent an attempt to determine whether a constant ratio of small to large nuclei is maintained by a plasmodium after its formation, or whether the nuclear fusion which is expected to occur during the slime mould life cycle might occur progressively during the time when a plasmodium has achieved macroscopic size. An attempt was made to characterize the infrequent changes in nuclear size ratio which occur, and to look for information as to whether or not the plasmodium possesses some means of regulating the ploidy level of its nuclear population.

\section{METHODS}

Organisms. Plasmodia were maintained on GPY/5 agar (glucose, peptone, yeast extract) spread with Aerobacter aerogenes (N. Kerr, I96I) and serially subcultured to fresh media at 2 day intervals. Each plasmodial stock was clonally derived from the progeny of a single amoeba. Two strains of a single isolate of Didymium nigripes were studied: c6 wild type (N. Kerr, 1965) and Y 2-1, a yellow mutant isolated on actidione agar (N. Kerr \& Waxlax, 1968b).

Determination of nuclear size. Nuclear size was determined using a Zeiss GFL phase contrast microscope with a $63 \mathrm{X}$ neofiuor objective and $12 \cdot 5 \mathrm{X}$ oculars. Smear preparations were made by placing small pieces of plasmodium on a slide in a drop of glycerine containing aceto-orcein, adding a coverslip, and disrupting the plasmodium by pressing gently on the coverslip. Nuclear size in young plasmodia was determined by observing living plasmodia growing on thin agar which had been transferred to a slide, covered with a coverslip, and sealed.

When nuclear size ratios in macroscopic plasmodia were determined, the size of 500 nuclei was determined and the number of small nuclei per 500 was recorded. No attempt was made to assign ploidy levels to extraordinarily large nuclei. Since within a plasmodium the mixing of contents occurs very rapidly (N. Kerr \& Waxlax, 1968 a), the nuclear population of small pieces of the plasmodium should be representative of the whole. Five hundred was chosen as the sample size because it was found that, with this size sample, counts from different portions of the same plasmodium duplicated each other.

Fusion of plasmodia. Plasmodia were fused by placing equal-sized pieces of two different plasmodia growing on agar adjacent to each other and next to a shelf of agar of the same thickness (see N. Kerr, 1965). During a period of 3 to $4 \mathrm{~h}$. such plasmodia migrate to the shelf of agar, come in contact with each other, and fuse.

Preparation of microplasmodia. Microplasmodia (small pieces of plasmodium) were prepared by scraping a plasmodium from the plate of agar, suspending it in $0.0 \mathrm{I} M$ 
pH 6.5 phosphate buffer (SS), homogenizing the suspension with a Vortex Junior mixer for $30 \mathrm{sec}$. and then allowing the suspension to stand for $30 \mathrm{~min}$. During this time the larger fragments of plasmodium settle to the bottom, and small pieces (many containing single nuclei) can be removed from the top of the suspension and plated on agar with Aerobacter aerogenes. After 2 to 3 days such microplasmodia grow to macroscopic size and can be isolated and subcultured in the same manner as other plasmodia.

\section{RESULTS}

\section{Changes in nuclear size composition of macroscopic plasmodia}

The proportion of small nuclei present in a plasmodial nuclear population was determined at weekly intervals in ten clonally derived plasmodia (plasmodia derived originally from a single amoeba) from each of two strains - the $\mathrm{c} 6$ wild type and the Y 2-I mutant. Representative data from three clones of each strain are shown in Table I.

Table I. Number of small nuclei observed in clones of plasmodia of Didymium nigripes strains $C 6$ and $Y 2-I$

In each observation, total number of nuclei counted was 500 . The $\mathrm{c} 6$ plasmodia were examined for two months and the mutant plasmodia (Y 2-I) for 100 days. The statistical significance of the observed changes was determined by using a $2 \times 2$ contingency table. Statistically significant changes $(P=0.01)$ are denoted by an asterisk.

\begin{tabular}{|c|c|c|c|c|c|c|c|c|}
\hline & & & Age & ays afte & ore gern & tion & & \\
\hline & $\mathrm{II}-\mathrm{I} 2$ & $17-18$ & $27-29$ & $35-36$ & $4 I-43$ & $4^{8-49}$ & $57-58$ & 63 \\
\hline Plasmodia & clone & & & & & & & \\
\hline c6-I & 369 & 369 & 388 & 390 & 397 & 395 & 398 & - \\
\hline c6-7 & 361 & 372 & * 435 & 414 & 410 & 416 & 417 & 417 \\
\hline C6-10 & 431 & 438 & $* 384$ & 404 & ${ }^{*} 468$ & 466 & $46 I$ & 466 \\
\hline Y 2-I A & 463 & $45 \mathrm{I}$ & $* 387$ & $* 307$ & $* 35 \mathrm{I}$ & $*_{40 I}$ & 404 & 435 \\
\hline Y 2-I B & 123 & $*_{404}$ & ${ }^{*} 136$ & $* 350$ & *395 & 399 & 370 & 397 \\
\hline Y 2-I F & 418 & $* 292$ & * I96 & $* 137$ & *353 & *303 & $*_{3} 68$ & *225 \\
\hline
\end{tabular}

If no change in ploidy level were occurring in the plasmodium, one would expect to find relatively little change in the proportion of small nuclei over extended periods of time. In this respect, $\mathrm{c} 6$ plasmodia showed very few changes of statistical significance: four of the ten plasmodia showed no changes at all and three more underwent only one change. One plasmodium behaved differently and demonstrated changes of nuclear size ratio at almost every observation. In general, $\mathrm{c} 6$ plasmodia did not undergo significant changes of ploidy level after 4 weeks of age. In contrast, most of the yellow clones exhibited changes in the nuclear size ratio at almost every observation. A summary of the changes in nuclear size in all 20 clones is included in Table 2.

There was no trend in the direction of significant changes, i.e. to more large nuclei or to more small nuclei. Of 13 changes detected in $\mathrm{c} 6$, eight resulted in more large nuclei and five in more small nuclei. Of 60 changes in Y 2-I clones, 32 led to more large nuclei and 28 led to more small nuclei. Within a given plasmodium, both continuation of a trend, e.g. to even more large nuclei, and alteration of direction of change, were noted on successive observations. 
In order to determine whether the observed long-term stability of nuclear size ratio is a property of the $\mathrm{c} 6$ strain or was simply due to the fact that the previously selected clones possessed a majority of small nuclei, a second series of c6 clones was isolated which showed three types of nuclear size ratio, i.e. mainly large, mainly small, and an appreciable proportion of both large and small (designated as mixed). In general, c 6 clones, regardless of initial nuclear size composition, showed less tendency to undergo changes in nuclear size ratio than did Y 2-I clones (Table 3).

\section{Table 2. Number of plasmodia showing changes in nuclear size ratios}

Table records number of plasmodia showing change in nuclear size ratio/total number of plasmodia examined $(P=0.01)$.

Age of plasmodia (weeks)

$\begin{array}{lccccccc}\text { Strain } & 3 & 4 & 5 & 6 & 7 & 8 & 9 \\ \text { C6 } & 1 / 9 & 5 / 10 & 2 / 7 & 3 / 7 & 0 / 9 & 1 / 9 & 1 / 7 \\ \text { Y2-1 } & 7 / 10 & 7 / 10 & 7 / 10 & 7 / 10 & 5 / 10 & 4 / 10 & 6 / 10\end{array}$

Table 3. c6 plasmodia showing changes in nuclear size ratios

Table records number of plasmodia showing changes in nuclear size ratio/total number of plasmodia examined $(P=0.01)$.

\begin{tabular}{lcccccc}
\multicolumn{7}{c}{ Age of plasmodia (weeks) } \\
Strain & I & 2 & 3 & 4 & 5 & 6 \\
Mainly small nuclei & $\mathrm{I} / 3$ & $\mathrm{I} / 2$ & $2 / 3$ & $\mathrm{I} / 3$ & $0 / \mathbf{1}$ & $0 / \mathrm{I}$ \\
Mainly large nuclei & $2 / 3$ & $3 / 3$ & $2 / 3$ & $0 / 3$ & $0 / 3$ & $0 / 3$ \\
Mixed & $\mathrm{I} / 4$ & $4 / 4$ & $\mathrm{I} / 4$ & $0 / 4$ & $0 / 3$ & $0 / 3$ \\
Total & $4 / 10$ & $8 / 9$ & $5 / 10$ & $1 / 10$ & $0 / 7$ & $0 / 7$
\end{tabular}

\section{Origin of large nuclei in the nuclear population}

A previous study (S. Kerr, $1968 a, b$ ) has shown that in populations of amoebae and in populations of young plasmodia derived from these amoeboid populations the proportion of small nuclei did not differ significantly. However, macroscopic plasmodia often possessed more large nuclei than the populations of amoebae from which they were derived. Occasional fortunate observations made with the phase contrast microscope revealed two methods by which large nuclei are formed: (i) fusion of two nuclei during interphase and (ii) failure of mitotic daughter nuclei to separate after telophase, with subsequent fusion. Large nuclei formed by both methods remained large throughout subsequent divisions, yielding large daughter nuclei.

The rate of formation of large nuclei in young plasmodia (4 to I6 nuclei) was calculated in populations of cells 12 to $30 \mathrm{~h}$. after they had been plated on agar following incubation procedures which give the largest numbers of plasmodia (N. Kerr, I96I). Cells were plated at densities such that secondary fusion of several plasmodia could not occur and account for the mixture of nuclear sizes. Two media were used: GPY $/ 5$, which gives a nuclear generation time of 4 to $6 \mathrm{~h}$., and a phosphate-buffered minimal media (SS; S. Kerr, I967) which gives a nuclear generation time of 6 to $8 \mathrm{~h}$. Of II25 plasmodia observed, 84 plasmodia contained both large and small nuclei.

The rate of formation of large nuclei was calculated in the following manner. Nuclear generations were defined such that generation ${ }_{0}$ was from plasmodial dif- 
ferentiation until division to the two-nucleate stage, generation ${ }_{1}$ was the two-nucleate stage until the second division, generation ${ }_{2}$ was the four-nucleate stage until the third division, etc. The simplest hypothesis explaining the generation of origin of large nuclei was accepted for each mixed plasmodium observed. For example, if one large and six small nuclei were present, only one hypothesis could explain this change of

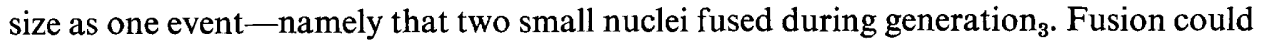
have occurred either between interphase nuclei or as a failure of mitosis but the generation of change would remain the same. Using this system, size change which occurred

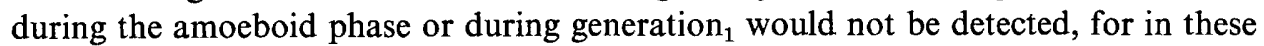
cases all resulting nuclei would be of uniform size.

On the medium which resulted in the longer generation time (SS), the observed rate of change per generation was higher (Table 4). In seven cases mixed plasmodia were observed which could be explained only by assuming that a large nucleus had split into two smaller ones. These data are not included in Table 4.

Table 4. Rate of formation of large nuclei in young plasmodia

$\begin{array}{lccccc}\text { Growth medium } & \text { Generation } & \begin{array}{c}\text { Number of } \\ \text { plasmodia } \\ \text { observed }\end{array} & \begin{array}{c}\text { Number of } \\ \text { plasmodia } \\ \text { with } \\ \text { mixed } \\ \text { nuclear } \\ \text { size }\end{array} & \begin{array}{c}\text { Number of } \\ \text { nuclear } \\ \text { generations } \\ \text { elapsed }\end{array} & \begin{array}{c}\text { Rate of } \\ \text { change per } \\ \text { nuclear } \\ \text { generation }\end{array} \\ \text { GPY/5 } & 2 & 293 & 14 & 2240 & 6.3 \times 10^{-3} \\ \text { GPY/5 } & 3 & 185 & 6 & 2136 & 2 \cdot 8 \times 10^{-3} \\ \text { Total } & 4 & 82 & 5 & 1312 & 3.8 \times 10^{-3} \\ \text { SS } & 2-4 & 560 & 25 & 5688 & 4.4 \times 10^{-3} \\ \text { SS } & 2 & 304 & 25 & 2224 & 1 \mathrm{I} \cdot 2 \times 10^{-3} \\ \text { SS } & 3 & 161 & 19 & 2016 & 9 \cdot 4 \times 10^{-3} \\ \text { Total } & 4 & 91 & 8 & 1456 & 5 \cdot 5 \times 10^{-3} \\ & 2-4 & 558 & 52 & 5696 & 9 \cdot 1 \times 10^{-3}\end{array}$

The second column shows the generation in which the large nuclei were estimated to have been formed first.

\section{Control of nuclear size ratio}

Several approaches were used in an attempt to determine whether a control mechanism exists in the plasmodium so that the nuclear size ratio is maintained at a fairly constant level.

Behaviour of microplasmodia. In a preliminary study, ten microplasmodia were isolated from each of three parental clones and their nuclear size ratios were compared to the ratios of their respective parents. Derivatives of one parental clone showed no significant difference in nuclear size ratios from the parent. Four and five of the isolated microplasmodia of the other clones differed from the parents after a 2 day period of time.

A second series of 12 microplasmodia were selected which differed from the nuclear size ratio of the parental clones. The ratios in these microplasmodia were followed for I 5 to 18 days to see if they would show any tendency to revert to the parental nuclear size ratio. Three of these microplasmodial lines underwent no change of ratio; six underwent a change of ratio but did not achieve a ratio like that of the parent; and three reverted to a ratio like that of their parents. Thus there seems to be no pronounced tendency for a given plasmodial stock to regulate back to a specific nuclear size ratio. 
Fusions of plasmodia with unlike ratios. Further attempts were made to determine whether or not maintenance of ploidy level and changes in ploidy level might be under some type of cytoplasmic control. Microplasmodia of $\mathrm{c} 6$ were isolated which showed three distinctive types of nuclear ratios: mainly small, mainly large, and an approximately equal number of small and large nuclei(mixed). These microplasmodia were grown to macroscopic size and then pieces of approximately equal size were fused with other pieces containing the same and different nuclear ratios in all possible combinations. Nuclear size ratios of the plasmodia derived from the fusion were determined $\mathrm{I}$ to 3 days later. Similar fusions were performed with clonally derived plasmodia. No difference in behaviour was found between plasmodia of these two origins.

Table 5. Fusions between plasmodia of different nuclear size ratios

Type of fusion $\quad \begin{gathered}\text { Number of } \\ \text { fusions }\end{gathered} \begin{gathered}\text { Number of } \\ \text { changes in } \\ \text { nuclear } \\ \text { size ratio } \quad \text { Direction of change }\end{gathered}$

(a) Controls (two pieces of the same plasmodium)

$\begin{array}{lrrr}\text { Mainly large } & 8 & 0 & - \\ \text { Mainly small } & 8 & \text { I } & \text { More small } \\ \text { Mixed } & \text { Io } & 9 & \text { More small }\end{array}$

(b) Fusions between clones

$\begin{array}{lrrl}\text { Large + large } & 5 & 0 & - \\ \text { Small + small } & 5 & 3 & \text { More small } \\ \text { Mixed +mixed } & 7 & 4 & \text { More small } \\ \text { Large + small } & \text { I2 } & \text { I2 } & \text { More small } \\ \text { Large+mixed } & 13 & 13 & \text { More small } \\ \text { Small + mixed } & \text { I6 } & \text { 13 } & \text { II More small } \\ & & & \text { 2 More large }\end{array}$

Where there was a significant change in ratio $(P=0.01)$ the direction of the change in nuclear size is shown in the last column.

The nuclear size ratios resulting from 84 such fusions were compared to a mean value derived from the parental stocks by means of a $2 \times 2$ contingency table (Table 5 ). The behaviour of plasmodia derived from such fusions was extremely consistent. Ploidy level changes generally occurred whenever one of the parental plasmodia contained an appreciable proportion of small nuclei (i.e. plasmodia characterized as either small or mixed) unless a given clone was being fused to a piece of itself. Of 55 changes in nuclear size ratio, all but two resulted in more small nuclei. In these two cases the 'small' parent for the fusions was the same clone.

Fusions of $C 6$ and $Y 2-1$ plasmodia. Clonally derived plasmodia of the $\mathrm{C} 6$ strain were fused with clonally derived plasmodia of the Y 2-I strain. Such 'heteroplasmodia' were examined weekly for 5 weeks in an attempt to determine whether they exhibited behavioural characteristics of either of the parental strains. These heteroplasmodia were compared both to their parents and to compiled data from other clones (Table 6).

In this case, 'heteroplasmodia' showed behavioural traits somewhat intermediate to the two parental types. The reduction in the number of ploidy level changes after a month of growth was reminiscent of the behaviour of the $\mathrm{c} 6$ whereas the percentage and magnitude of the changes observed before this time was more like the behaviour observed in the yellow mutant clones. 
The C6+Y2-I heteroplasmodia were characterized by loss of pigmentation a few hours after formation. After the 5 weeks of observation the composition of the plasmodia was checked by two methods for the continued presence of both parental types. Microplasmodia were isolated and incubated until colour could be determined. The heteroplasmodia were also allowed to fruit, the resulting spores were plated so they would give rise to clones, and then the colour of the resulting amoeboid clones was scored. With the exception of one plasmodium which had become yellow during the 5 weeks (and which yielded only yellow progeny), both parental types were recovered from all the heteroplasmodia.

Table 6. $C 6+Y 2-I$ heteroplasmodia ploidy level changes

Table records number of plasmodia showing changes in nuclear size ratio/total number of plasmodia examined $(P=0.01)$.

\section{Type of plasmodia}

All c6

All Y 2-I

C6 parents

Y 2-I parents

Fusion plasmodia; equal

parental contribution

Fusion plasmodia;

I/3 Y 2-I; 2/3 C 6

Fusion plasmodia;

$2 / 3$ Y $2-1 ; 1 / 3$ c 6

Total of fusions

\begin{tabular}{ccccc}
\multicolumn{5}{c}{ Age of plasmodia (weeks) } \\
$\overbrace{\mathrm{I}}^{2}$ & 3 & 4 & 5 \\
$4 / 10$ & $8 / 9$ & $6 / 19$ & $6 / 20$ & $2 / 14$ \\
$\mathrm{I} / 2$ & $10 / \mathrm{I} 2$ & $10 / 12$ & $8 / 12$ & $8 / 12$ \\
$\mathrm{I} / 3$ & $0 / 3$ & $0 / 3$ & $0 / 3$ & $0 / 3$ \\
$\mathrm{I} / 2$ & $2 / 2$ & $2 / 2$ & $1 / 2$ & $1 / 2$ \\
$4 / 6$ & $4 / 6$ & $4 / 6$ & $4 / 6$ & $0 / 6$ \\
$\mathrm{I} / 2$ & $2 / 2$ & - & - & - \\
$\mathrm{I} / 2$ & $\mathrm{I} / 2$ & $2 / 2$ & $1 / 2$ & $0 / 2$ \\
$6 / 10$ & $7 / 10$ & $6 / 8$ & $5 / 8$ & $0 / 8$
\end{tabular}

\section{DISCUSSION}

\section{Mixture of nuclear sizes in plasmodia}

The mixture of nuclear sizes as reported here for Didymium nigripes is not a new observation for true slime moulds. Ross (I966), Koevenig \& Jackson (I966) and Guttes \& Guttes (1969) have recently reported several sizes of nuclei which represent different ploidy levels in the plasmodium of Physarum polycephalum. In addition, Ross reported several ploidy levels in amoebae of Badhamia curtisii. Therrien (1966) used microspectrophotometry to measure the DNA content of individual nuclei of a single plasmodium of the isolate of $D$. nigripes used in the current investigation and found that these nuclei may have vastly differing amounts of DNA, even though all the nuclei in a single plasmodium should be in the same phase of the cell cycle at a given time. Counts of the number of chromosomes present also show that nuclei of several ploidy levels are present (S. Kerr, I $968 a, b$ ). The present study reported only the proportion of nuclei of a certain size class which were present. Very rarely, nuclei of smaller diameter were encountered: routinely the 'large' nuclei varied greatly in nuclear diameter.

In the light of both chromosome counts and Therrien's calculation of DNA content, it seems expedient at the present time not to assign small nuclei to a haploid class, but simply to regard nuclei of various sizes as several different multiples of an, as yet, undetermined haploid number of chromosomes. 
Nuclear size fluctuations

Observations dealing with maintenance of nuclear size ratios or change in these ratios over a period of time have not been reported previously for the true slime moulds. The calculated rate of formation of large nuclei in populations of young plasmodia is great enough to account for all of the changes in nuclear size ratio which have been observed. After examining the data collected in this study, it is rather difficult to construct a model for control of nuclear size ratios. If formation of large nuclei continued to occur at the initial rate, plasmodia would soon be composed solely of large nuclei. Although indirect evidence for formation of small nuclei from large nuclei was obtained, this rate was much lower and could not account for the maintenance of a constant ratio of small to large nuclei. During sporulation large-scale destruction of nuclei has been observed: some of the nuclei became enclosed in vacuoles and were gradually destroyed. Nuclear destruction of this type is rarely observed in a growing c6 plasmodium, however, and so it seems unreasonable to assume that a plasmodium maintains a fairly constant nuclear size ratio by large scale destruction of its large nuclei.

Yellow plasmodia underwent massive fluctuations in size classes and at the same time differed from $\mathrm{C} 6$ in other ways. Yellow plasmodia were less hardy and underwent an ageing process which was characterized by loss of the ability to sporulate, loss of the ability to fuse with other plasmodia and, finally, loss of the ability to grow (N. Kerr \& Waxlax, I968 b). Smear preparations of yellow plasmodia undergoing division showed that most extremely large and occasional large nuclei failed to divide at the same time as the small nuclei were dividing. Yellow plasmodia contained degenerating nuclei in vacuoles and also possessed a much greater proportion of polyploid nuclei, and on occasions a majority of the nuclei have seemed to be of extraordinary diameter. However, there was no correlation between the onset of ageing symptoms, accumulation of polyploid nuclei, and changes in the nuclear size ratio.

\section{Is nuclear size controlled?}

One hypothesis which must be examined is that a plasmodium may be able to control the proportions of nuclei in its various size classes and thus maintain what seems to be an optimum distribution of sizes. In this case one would expect a clonally derived plasmodium not only to maintain a fairly constant distribution of sizes in its nuclear population, but also for tiny pieces of this plasmodium to achieve the same ratio. In cases where microplasmodia were isolated and nuclear size ratios studied, there was no indication that maintenance of a special ratio was occurring.

An alternate hypothesis might be that a fairly constant nuclear size ratio is maintained simply by divison of all existing nuclei and that changes in ratio are precipitated only by sampling errors during subculture. Although this hypothesis might explain the behaviour of microplasmodia, several types of observations suggest that this hypothesis in untenable. Rapid mixing of all cytoplasmic components within a plasmodium has been reported within the isolate studied using both stained and radioactively labelled plasmodia (N. Kerr \& Waxlax, I968a). Samples I mm. ${ }^{2}$ contain several thousand nuclei and nuclear ratios from various portions of the same plasmodium in samples of this size are virtually identical. Subculture blocks $1 \mathrm{~cm} .^{2}$ would then be 
expected to contain representative distribution of the nuclear sizes present. In most cases a constant ratio was maintained by this subculture technique.

If the nuclear ratio remains constant through a passive means, then it becomes difficult to explain several observations: $(a)$ the relatively constant rate of formation of large nuclei in young plasmodia, $(b)$ the results from fusions between pieces of plasmodia with different size ratios, in which the resulting size ratio was unlike the mean derived from the two parental ratios and yet all nuclei present seemed to divide synchronously, and $(c)$ the rather extensive alterations in nuclear size ratio in yellow plasmodia.

The fact that ploidy level changes do occur is particularly interesting since critical cytological and genetic evidence of standard sexual processes are lacking in this organism. Perhaps the behaviour observed can be considered analogous to parasexual processes described by Pontecorvo (1958) in the fungus Aspergillus nidulans. The sequence of events described by Pontecorvo involved rare fusion of haploid nuclei to form a diploid nucleus and subsequent segregation of recombinant nuclei with eventual haploidization. The occasional fusion of nuclei in Didymium nigripes and variability of chromosome number (S. Kerr, I968 a,b) and DNA content (Therrien, I966) could be cited as evidence for diploidization followed by progressive haploidization. A search for parasexual processes by genetic analysis has not yet been attempted in D. nigripes: at the same time the cytological observations reported here suggest that parasexuality should not be excluded as a possible genetic mechanism in the true slime moulds.

I wish to thank Dr Norman Kerr for the use of his laboratory and for helpful discussion on the preparation of this paper. Parts of this work were supported by two grants to Dr Norman Kerr-the Biomedical Research Fund of the Graduate School, University of Minnesota, and N.I.H. Institute of Allergy and Infectious Disease grant AI-0552I.

\section{REFERENCES}

Gutres, E. \& GutTes, S. (1969). Replication of nucleolus-associated DNA during $\mathrm{G}_{2}$ Phase in Physarum polycephalum. Journal of Cell Biology 43, 229-236.

KERR, N. (196I). A study of plasmodium formation by the true slime mould Didymium nigripes. Experimental Cell Research 23, 603-6I I.

KERR, N. (1965). Disappearance of a genetic marker from a cytoplasmic hybrid plasmodium of a true slime mould. Science, New York 147, I586-I 588.

KerR, N. \& WAXLAX, J. (1968a). The fusion of plasmodia of a true slime mould grown on various nutrients and the mixing of fused plasmodia. Transactions of the American Microscopical Society $87,197-200$.

KerR, N. \& Waxlax, J. (1968b). A yellow variant of the Eumycetozoan Didymium nigripes which exhibits ageing. Journal of Experimental Zoology I68, 35I-36I.

KERR, S. (1967). A comparative study of mitosis in amoebae and plasmodia of the true slime mould Didymium nigripes. Journal of Protozoology $\mathbf{4} 4,439-445$.

KERR, S. (1968a). Cytological observations on plasmodial differentiation in the true slime mould Didymium nigripes. $\mathrm{PhD}$. Dissertation, University of Minnesota.

KERR, S. (I968b). Ploidy level in the true slime mould Didymium nigripes. Journal of General Microbiology 53, 9-15.

Kozvenig, J. L. \& JACKson, R. C. (1966). Plasmodial mitosis and polyploidy in the Myxomycete Physarum polycephalum. Mycologia 53, 662-667. 
NygaARd, O. F., Guttes, S. \& Rusch, H. P. (1960). Nucleic acid metabolism in a slime mould with synchronous mitosis. Biochimica et Biophysica Acta 38, 298-306.

PonTECorvo, G. (1958). Trends in Genetic Analysis, pp. I I9-I24. New York: Columbia University Press.

Ross, I. K. (1966). Chromosome numbers in pure and gross cultures of Myxomycetes. American Journal of Botany 53, 7I 2-7 I8.

Therrien, C. D. (1966). Microspectrophotometric measurement of nuclear deoxyribonucleic acid content in two myxomycetes. Canadian Journal of Botany 44, 1667-1675. 